\title{
Dihydroflavonol 4-reductase activity is associated with the intensity of flower colors in delphinium
}

\author{
Natsuki Miyagawa', Taira Miyahara', Mitsutoshi Okamoto², Yukio Hirose², \\ Kimitoshi Sakaguchi ${ }^{3}$, Shoji Hatano ${ }^{3}$, Yoshihiro Ozeki ${ }^{1, *}$ \\ ${ }^{1}$ Department of Biotechnology, Tokyo University of Agriculture and Technology, Koganei, Tokyo 184-8588, Japan; \\ ${ }^{2}$ Department of Agricultural Research, Ehime Research Institute of Agriculture, Forestry and Fisheries, Matsuyama, Ehime \\ 799-2405, Japan; ${ }^{3}$ Miyoshi \& Co., Ltd., R\&D Center, Hokuto, Yamanashi 408-0041, Japan \\ *E-mail: ozeky@cc.tuat.ac.jp Tel \& Fax: +81-42-388-7239
}

Received April 21, 2015; accepted July 2, 2015 (Edited by T. Aoki)

\begin{abstract}
Flower color intensity is largely determined by the amount of accumulated anthocyanins. Delphinium flowers show a wide range of colors from pale pink to deep orange to red to dark blue. Here, we demonstrated that the level of anthocyanin accumulation in dark blue, orange and red varieties was higher than in pale blue and pale pink varieties. Since dihydroflavonol 4-reductase (DFR) is a key enzyme in anthocyanin biosynthesis and accumulation in plants, we investigated the relationship between flower color intensity and the level of DFR gene expression. Six delphinium varieties with different flower colors were analyzed. Varieties that accumulated relatively high levels of anthocyanin also had high levels of DFR expression and enzyme activity in crude protein extracts. By contrast, DFR expression and activity was low in varieties with low anthocyanin accumulation. Alignment of DFR amino acid sequences in the six varieties showed the presence of two types, termed DgDFR and DnDFR. Recombinant DgDFR and DnDFR proteins had similar substrate specificities, but the kinetic turnover rate of the DnDFR enzyme was higher than that of DgDFR. We conclude that DFR expression level is closely correlated with flower color intensity and that DFR is an important factor that determines anthocyanin accumulation and delphinium flower color intensity.
\end{abstract}

Key words: Anthocyanin, delphinium, dihydroflavonol 4-reductase.

The variability of flower coloration in plants is determined by the accumulation of pigments such as anthocyanins. Anthocyanins are synthesized and accumulated in many plant species and are responsible for a wide range of flower colors, such as red, pink, orange, purple and blue. There is a close relationship between color and structure of the anthocyanin aglycone (anthocyanidin) (Nishihara and Nakatsuka 2011; Tanaka and Burglira 2013). The biosynthetic pathway for the anthocyanidins pelargonidin $(\mathrm{Pg})$, cyanidin (Cy) and delphinidin (Dp) has been shown to start with phenylalanine and involve production of intermediates, for example, dihydroflavonols such as dihydrokaempferol (DHK), dihydroquercetin (DHQ), and dihydromyricetin (DHM), and leucoanthocyanidins such as leucopelargonidin (LPg), leucocyanidin (LCy), and leucodelphinidin (LDp). The biosynthesis of these compounds is mediated by the enzymes flavonoid $3^{\prime}$-hydroxylase $\left(\mathrm{F} 3^{\prime} \mathrm{H}\right)$, flavonoid $3^{\prime}, 5^{\prime}$-hydroxylase $\left(\mathrm{F}^{\prime} 5^{\prime} \mathrm{H}\right)$, dihydroflavonol 4-reductase (DFR) and anthocyanidin synthase. Although delphiniums are most familiar as blue flowers, varieties bearing pale pink, orange and red flowers have been developed through breeding or by screening wild plant populations. Since red delphiniums can have a high value in horticulture, production of varieties showing different intensities and tones of red is likely to be commercially important. Elucidation of the mechanism of coloration can be used to produce new color varieties not only by breeding strategies using DNA markers, but also through use of transgenic technology such as inter-species transfer of the appropriate genes in the case of blue carnations and roses and of red petunia (Katsumoto et al. 2007; Meyer et al. 1987; Mol et al. 1999; Tanaka

Abbreviations: CRD, Delphinium cardinale; Cy, cyanidin; DAD, diode array detector; DFR, dihydroflavonol 4-reductase; DgDFR, Delphinium grandiflorum DFR; DHK, dihydrokaempferol; DHM, dihydromyricetin; DHQ, dihydroquercetin; DnDFR, Delphinium nudicaule DFR; Dp, delphinidin; ESI-MS, electrospray ionization-mass spectrometry; F3' $5^{\prime} \mathrm{H}$, flavonoid 3',5'-hydroxylase; F3' $\mathrm{H}$, flavonoid 3'-hydroxylase; G6P, glucose-6-phosphate; G6PDH, glucose-6-phosphate dehydrogenase from Leuconostoc mesenteroides; GB, $\mathrm{F}_{1}$ Super Grand Blue; GST, glutathione Stransferase; HPLC, high-performance liquid chromatography; KPi, potassium phosphate buffer; LCy, leucocyanidin; LDp, leucodelphinidin; LPg, leucopelargonidin; NDC, Delphinium nudicaule; PB, $\mathrm{F}_{1}$ Super Platinum Blue; Pg, pelargonidin; Qr, quercetin; qRT-PCR, quantitative RT-PCR; SHP, $\mathrm{F}_{1}$ Super Happy Pink; SKH, Sakurahime; TFA, trifluoroacetic acid.

This article can be found at http://www.jspcmb.jp/

Published online August 18, 2015 

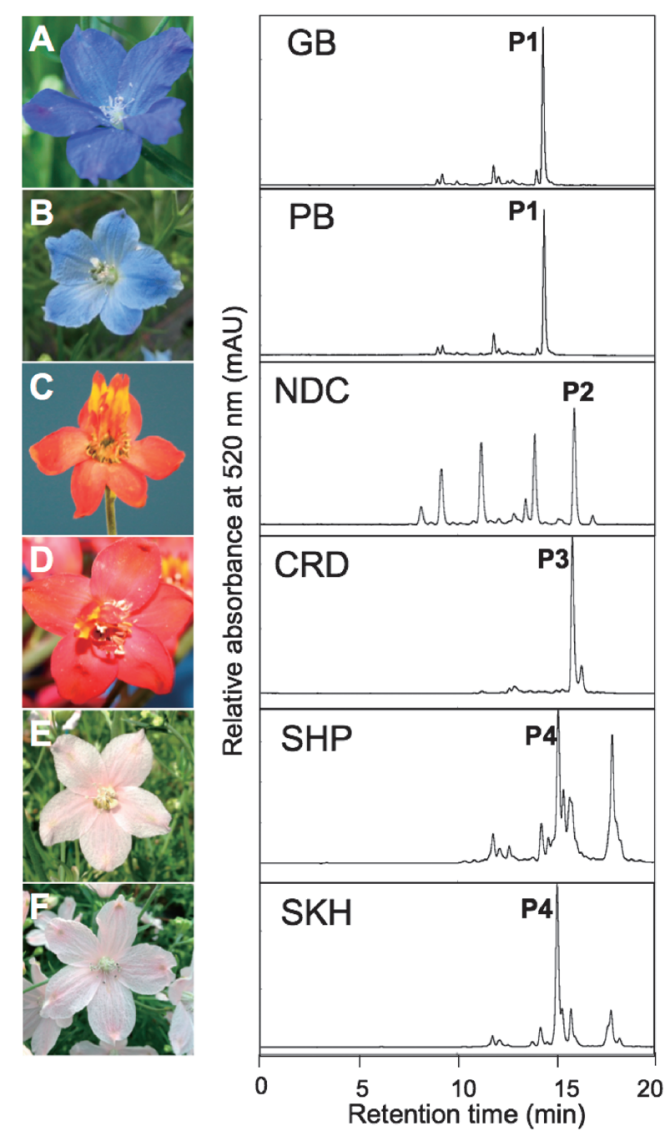

Figure 1. Flower colors in six delphinium varieties and highperformance liquid chromatography (HPLC) analysis to determine their main anthocyanins. (A) ' $F_{1}$ Super Grand Blue (GB)', (B) ' $F_{1}$ Super Platinum Blue (PB)', (C) D. nudicaule (NDC), (D) D. cardinale (CRD), (E) ' $\mathrm{F}_{1}$ Super Happy Pink (SHP)' and (F) 'Sakurahime (SKH)', P1, cyanodelphin; P2, Pg 3-O-(6-malonyl)glucoside; P3, Pg 3-O-(6malonyl)glucoside-7-O-(6-O-(4-O-(6-O-(p-hydroxybenzoyl)-glucosyl)oxybenzoyl)-glucoside); $\mathrm{P} 4, \mathrm{Pg}$ 3-O-rutinoside-7-O-(6-O-(4-O-(6-O( -hydroxybenzoyl)-glucosyl)-oxybenzoyl)-glucoside).

et al. 1998). To produce new red varieties, we need to fully describe the mechanisms responsible for flower colors in delphiniums. Recently, we showed that the factor determining whether a flower is blue or red is the $\mathrm{F}^{\prime} 5^{\prime} \mathrm{H}$ gene (Miyagawa et al. 2014). Delphinium varieties displaying blue hues in their flowers accumulate derivatives of the blue pigment Dp (Figure 1A, B). In our earlier study, we used three red flowered delphinium varieties. These varieties were found to have different mechanisms for repression of the $F 3^{\prime} 5^{\prime} H$ gene that is required to synthesize the blue pigment $\mathrm{Dp}$. As a consequence of repression of $F 3^{\prime} 5^{\prime} H$, the plants synthesize and accumulate derivatives of the red pigment $\mathrm{Pg}$ rather than of $\mathrm{Dp}$ in their sepals. Although red varieties accumulate the aglycone Pg, nevertheless, they can show clearly different phenotypes: garden varieties belonging to Delphinium grandiflorum tend to be pale pink, whereas the wild species $D$. nudicaule (NDC) and D. cardinale (CRD) have dark orange and red flowers, respectively. In recent years, pale pink horticultural cultivars belonging to $D$. grandiflorum have been established; however, transfer of the dark orange and red phenotypes from 'NDC' and 'CRD' into D. grandiflorum (which has superior agronomic and ornamental traits) has not yet succeeded. At present, the mechanism that determines the intensity of color in delphiniums is unclear. To elucidate this mechanism, we investigated the major accumulated anthocyanidin molecules and the amounts of anthocyanins in six varieties of delphinium showing different flower coloration.

The six delphinium cultivars selected for this study are illustrated in Figure 1. The D. grandiflorum hybrid lines ' $\mathrm{F}_{1}$ Super Grand Blue (GB)', which has dark blue flowers, ' $\mathrm{F}_{1}$ Super Platinum Blue $(\mathrm{PB})$ ', which has pale blue flowers, and ' $F_{1}$ Super Happy Pink (SHP)', which has pale pink flowers, were obtained from Miyoshi Co., Ltd. The D. grandiflorum $\mathrm{F}_{1}$ hybrid variety 'Sakurahime (SKH)', which has pale pink flowers, and the wild species 'NDC', which has orange flowers, and 'CRD', which has red flowers, were a gift from the Ehime Research Institute of Agriculture, Forestry and Fisheries. For all six varieties, sepals at stage 3 or 4 from four different developmental stages (stage 1, bud; stage 2, beginning to bloom; stage 3, $80 \%$ open; stage 4 , fully open; see Supplementary Figure S1) were collected and immediately frozen in liquid nitrogen, and stored at $-80^{\circ} \mathrm{C}$ until use.

Purple-red, purple and blue varieties of delphinium have delphinidin 3-rutinoside, violdelphin and cyanodelphin, respectively, as the major anthocyanin (Hashimoto et al. 2002; Kondo et al. 1990, 1991). To determine whether the modifications contribute to color intensity, we performed a high-performance liquid chromatography (HPLC) analysis of the main anthocyanidin molecules in the six varieties. For these HPLC analyses, each frozen sample (3-5 pieces of stage 4 sepals) was soaked in $1 \mathrm{ml} 80 \%$ aqueous methanol containing $0.1 \%$ trifluoroacetic acid (TFA) for $4 \mathrm{~h}$ at $4^{\circ} \mathrm{C}$ to extract the pigments. The extracted pigments were separated by HPLC and retention times were compared to authentic pigments stored in our laboratory. The HPLC-diode array detector (DAD) analysis was carried out using a LaChromElite System (pump L-2130, column oven L-2300, diode array detector L-2450, Hitachi High-Technologies, Tokyo, Japan) equipped with a reversed-phase column of Handy ODS (i.d. $4.6 \mathrm{~mm} \times 250 \mathrm{~mm}$, Wako Pure Chemical Industries, Ltd., Osaka, Japan). Detection was performed at $520 \mathrm{~nm}$. Separation conditions involved linear gradient elution at a flow rate of $1 \mathrm{ml} \mathrm{min}^{-1}$ from 20 to $80 \%$ methanol in $0.1 \%$ aqueous TFA for $20 \mathrm{~min}$. The separated main anthocyanin fractions were retrieved and then subjected to electrospray ionization-mass spectrometry (ESI-MS) (AccuTOF MS, JMS-T100LC, JEOL, Tokyo, Japan).

Four peaks were identified in the analysis of the six varieties; these peaks are termed P1, P2, P3 and P4 
(Figure 1). Although there was a clear difference in floral color phenotype between the dark blue variety 'GB' and the pale blue variety 'PB' (Figure 1A, B), both had the same major anthocyanin (P1). ESI-MS analysis indicated that this was cyanodelphin. The 'NDC' variety, which has orange flowers (Figure 1C), had P2 as the major anthocyanin; ESI-MS analysis indicated that this was Pg 3-O-(6-malonyl)glucoside with maximum absorption at $510 \mathrm{~nm}$. The 'CRD' variety, which has red flowers (Figure 1D), had the P3 peak, while the pale pink varieties 'SKH' and 'SHP' (Figure 1E, F) had the P4 peak; ESI-MS indicated that these peaks were $\mathrm{Pg} 3-\mathrm{O}$-(6-malonyl)glucoside-7-O-(6-O-(4-O-(6-O-(p-hydroxybenzoyl)glucosyl)-oxybenzoyl)-glucoside) and Pg 3-O-rutinoside7-O-(6-O-(4-O-(6-O-( $p$-hydroxybenzoyl)-glucosyl)oxybenzoyl)-glucoside), respectively, with maximum absorption at $515 \mathrm{~nm}$. Differences in modification of the major anthocyanin caused differences in maximum absorption, and gave rise to the orange, red and pale pink coloration groups. Although there were clear phenotypic differences between the 'CRD' (red flowers) and 'SKH' and 'SHP' (pale pink flowers) varieties, the anthocyanin structures in the three varieties were similar; in particular, the 7-position of Pg was modified with the same residues that are believed to play an important role in determining flower color (Nishizaki et al. 2013). The HPLC analysis and ESI-MS analysis suggested that the structure of the main anthocyanin had less effect on color quality in varieties with red, orange and pale pink flowers.

Recent reports in anthurium and soybean have confirmed the strong correlation between color intensity and anthocyanin accumulation (Gopaulchan et al. 2014; Yan et al. 2014). To determine whether this effect also occurred in delphinium, we measured the amounts of anthocyanin in the sepals of the six varieties. For this analysis, sepals at stage 4 , the fresh weight of which had been measured, were soaked in $1 \mathrm{ml} 80 \%$ methanol containing $0.1 \%$ TFA for $4 \mathrm{~h}$ at $4^{\circ} \mathrm{C}$ to extract the pigments. The amount of extracted pigments was measured at $510 \mathrm{~nm}$ in red varieties and at $530 \mathrm{~nm}$ in blue varieties using a U-5100 spectrophotometer (Hitachi High-Technologies Co., Tokyo, Japan). The amount of anthocyanin was calculated by absorbance at 510 or $530 \mathrm{~nm}\left(\mathrm{~A}_{510}\right.$ or $\left.\mathrm{A}_{530}\right)$ per $\mathrm{g}$ fresh weight of sepals. Three independent samples were measured to provide average values and standard errors.

Anthocyanin levels in the dark blue variety 'GB', the orange species 'NDC' and the red species 'CRD' were 4-13 times higher than in the pale blue variety ' $\mathrm{PB}$ ' and the pale pink varieties 'SKH' and 'SHP' (Figure 2C). Thus, there was a clear relationship between anthocyanin accumulation and color intensity.

Previous studies on anthocyanin biosynthesis reported that activity of the anthocyanin biosynthetic enzyme
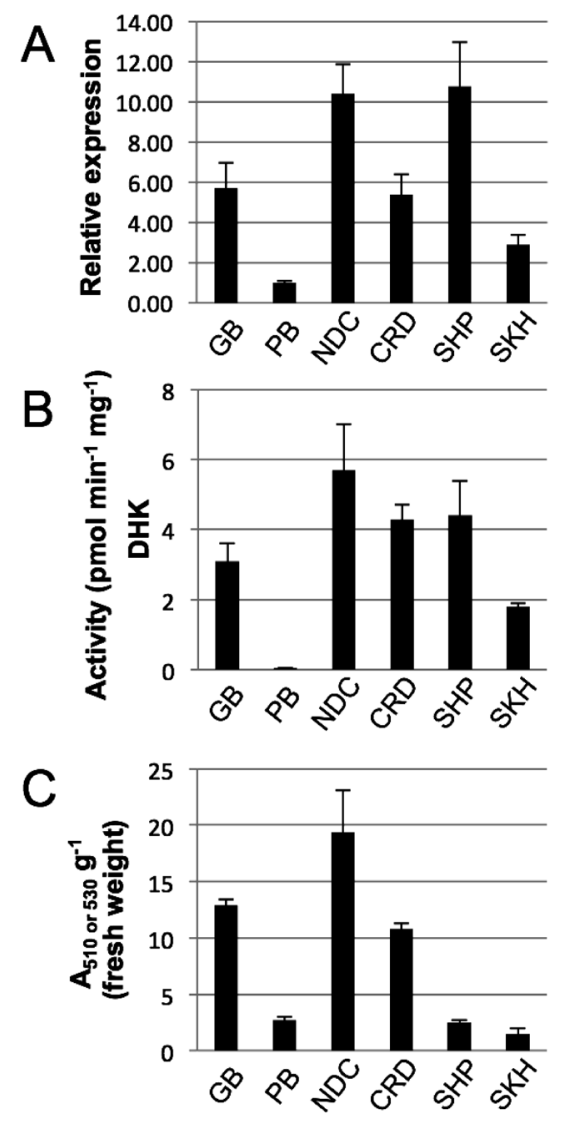

Figure 2. Comparison of the relative levels of DFR expression, DFR activity in crude protein extracts, and anthocyanin accumulation in sepals of six delphinium varieties. (A) The expression of DFR was normalized against Actin. RNAs were prepared from sepals and first strand cDNAs were synthesized; quantitative RT-PCR was performed using the DgDFR ATG and qRTDgDFR Rev primers. Actin was used as the reference gene for RNA extraction, cDNA synthesis and PCR amplification. The level of expression in 'PB' was arbitrarily set as 1 . The abbreviations for varietal names are as described in Figure 1. (B) DFR activity in crude protein extracts from sepals was quantified by measuring the rate of conversion of leucoanthocyanidin to anthocyanidin. Reaction products were separated by HPLC and the anthocyanidin peak areas were used to calculate the product amounts catalyzed by DFR. (C) Extracted pigments from the sepals were analyzed by spectrophotometry at $510 \mathrm{~nm}$ in red varieties and at $530 \mathrm{~nm}$ in blue varieties. The amount of anthocyanin was calculated by absorbance at 510 or $530 \mathrm{~nm}\left(A_{510}\right.$ or $\left.A_{530}\right)$ per g fresh weight of sepals. Relative percentages are shown in parentheses in the right panels.

dihydroflavonol 4-reductase (DFR) is an important rate-limiting step (Collette et al. 2004; Forkmann and Ruhnau 1987; Helariutta et al. 1993; Johnson et al. 1999; Mato et al. 2000). DFR is known to catalyze the reaction transforming dihydroflavonols (DHK, DHQ, and DHM) to leucoanthocyanidins (LPg, LCy, and LDp) using $\beta$-NADPH (Heller et al. 1985; Stafford and Lester $1982,1985)$. In anthurium and carnation, the levels of DFR transcripts in varieties with pale colored flowers or white varieties were clearly lower than in those with deeply colored flower varieties, indicating that the DFR step limits the metabolic production of anthocyanins (Collette et al. 2004; Mato et al. 2000). Additionally, in 
cymbidium, gerbera and petunia, DFR influences flower coloration through its substrate specificity as it shows a preference for catalyzed substrates biased toward the synthesis of catabolite species and thus determining the major anthocyanidin molecules (Forkmann and Ruhnau 1987; Helariutta et al. 1993; Johnson et al. 1999). To further elucidate the relationship between DFR activity and flower color intensity, we investigated DFR expression levels in delphinium sepals by measuring the amounts of mRNA transcripts; we also determined DFR enzyme activities in crude protein extracts and examined the properties of a recombinant DFR enzyme.

First, we isolated $\operatorname{Dg} D F R$ cDNA. Total RNAs were extracted from sepals of the pale blue variety 'PB' and first strand cDNAs were synthesized as described previously (Nishizaki et al. 2011). Two degenerate primers, DFRdg Fwd and DFRdg Rev (Supplementary Table S1), were designed using the sequences of DFR genes of other plant species. Degenerate RT-PCR using the first strand cDNAs as the template and with TaKaRa Ex Taq Hot Start Version (Takara Bio Inc, Ohtsu, Japan) was performed under the following conditions: 37 cycles of $10 \mathrm{~s}$ denaturation at $98^{\circ} \mathrm{C}, 30 \mathrm{~s}$ annealing at $45^{\circ} \mathrm{C}$, and $60 \mathrm{~s}$ extension at $72^{\circ} \mathrm{C}$. The nucleotide sequence of the initial DgDFR cDNA fragment obtained by degenerate PCR was used to design the DgDFR3RACE Fwd and DgDFR5RACE Rev primers (Supplementary Table S1). The $5^{\prime}$ and $3^{\prime}$ cDNA ends of DgDFR were obtained as described previously (Miyagawa et al. 2014). This cDNA sequence was used to design the DgDFR ATG and DgDFR STOP primers, and the qRTDgDFR Rev primer (Supplementary Table S1). To isolate DFR cDNAs from each of the six varieties, RT-PCR was performed using first strand cDNAs of stage 3 sepals (prepared as described above) as the template with Prime STAR GXL DNA Polymerase (Takara Bio) and DgDFR ATG and DgDFR STOP primers; amplification conditions were 35 cycles of $10 \mathrm{~s}$ denaturation at $96^{\circ} \mathrm{C}, 15 \mathrm{~s}$ annealing at $58^{\circ} \mathrm{C}$, and $15 \mathrm{~s}$ extension at $68^{\circ} \mathrm{C}$.

The DFR coding region was amplified from 'PB' sepals by PCR using the DgDFR ATG and DgDFR STOP primers; six independent Escherichia coli clones carrying DFR cDNA were generated and analyzed. All six nucleotide sequences were completely identical (GenBank accession number LC029437). As 'PB' is an $\mathrm{F}_{1}$ hybrid line, it was possible that the parental lines might have carried different $D F R$ alleles with different nucleotide sequences. However, we presume that 'PB' originated by cross-hybridization of parents with identical DFR alleles as only one cDNA sequence was present. Three independent cDNA clones from the $\mathrm{F}_{1}$ hybrid 'SHP' showed identical nucleotide sequences (accession number LC029440); similarly, three clones from 'SKH' were identical (accession number LC029441). On the other hand, the $F_{1}$ hybrid 'GB' yielded two cDNA fragments after PCR amplification using DgDFR ATG and DgDFR STOP primers: a 1,014bp fragment and a $1,083 \mathrm{bp}$ fragment. The nucleotide sequences of three independent clones for the $1,014 \mathrm{bp}$ fragment were analyzed; two had an identical sequence and lacked a stop codon inside (accession number LC029436); the third had a single nucleotide alteration at nucleotide position 691 (A to G) that caused a change from an asparagine residue to aspartate in the amino acid sequence. The nucleotide sequences of two clones for the $1,083 \mathrm{bp}$ fragment revealed they were identical; both had a $69 \mathrm{bp}$ insertion at nucleotide position 108 compared to the 1,014 bp fragment of 'GB'. This insertion generated a stop codon inside and we judged that it produced a pseudo DRF gene. For the quantitative RT-PCR (qRTPCR) analysis, we designed a primer, qRTDgDFR Rev, that corresponded to the nucleotide sequence from position 98 to $125 \mathrm{bp}$ of the $1,014 \mathrm{bp}$ fragment of 'GB'; this primer did not anneal to the long 1,083 bp fragment of 'GB' due to the insertion sequence, nor did it amplify a PCR product from the pseudo DFR gene.

The cDNA sequences of three independent clones derived from the orange flowered variety 'NDC' were identical (accession number LC029438); by contrast, three independent clones from the red flowered variety 'CRD' yielded two identical sequences (accession number LC229439) and one that showed two nucleotide sequence alterations: $\mathrm{T}$ to $\mathrm{C}$ at position $165 \mathrm{bp}$ and $\mathrm{T}$ to $\mathrm{A}$ at position $843 \mathrm{bp}$. Neither alteration caused an amino acid sequence change. The qRTDgDFR Rev primer sequence was designed to avoid these sequences.

Alignment of the DFR amino acid sequences derived from the cDNAs of each of the six varieties showed that they fell into two clear groups in terms of phylogenetic relationship (Supplementary Figure S2). The DFR amino acid sequences of $D$. grandiflorum 'GB', 'PB', 'SKH' and 'SHP' varieties belonged to one clade, while those of D. nudicaule (NDC) and D. cardinale (CRD) belonged another. This separation might be due to differences among the Delphinium species. We named the former group DgDFR and the latter group DnDFR. The DFR amino acid sequence of many plant species has been reported to contain a conserved asparagine residue at position 134, although Petunia hybrida has an aspartate residue at this location. A point mutation that causes a change from asparagine to leucine at position 134 in Gerbera hybrida DFR alters the anthocyanidin products from a mixture of Pg, Cy and Dp to only Pg in the petals of transgenic petunia flowers (Johnson et al. 2001). This implies that Gerbera hybrida DFR prefers DHK as a substrate to DHQ and DHM in transgenic petunia plants; however, the detailed kinetic properties of these substrates have yet to be confirmed. In the amino acid sequences of the six varieties of delphinium, an asparagine residue is present at the corresponding 
position 131 (Supplementary Figure S2A).

DFR expression levels in the six varieties were analyzed by qRT-PCR using first strand cDNAs as templates and the DgDFR ATG and qRTDgDFR Rev primers for DFR and the DpActinF and DpActinR primers for Actin (Supplementary Table S1). The amplification conditions were 36 cycles of $30 \mathrm{~s}$ denaturation at $95^{\circ} \mathrm{C}, 30 \mathrm{~s}$ annealing at $58^{\circ} \mathrm{C}$, and $30 \mathrm{~s}$ extension at $72^{\circ} \mathrm{C}$; the SYBR Premix Ex Taq (Takara Bio) and the DNA Engine Opticone 2 (BioRad Laboratories Inc, Hercules, CA, USA) were used. Actin was used as the reference gene for quantification of DgDFR expression.

DFR enzyme activities in crude protein extracts were determined by first grinding $0.2-0.5 \mathrm{~g}$ of stage 4 sepals into powder in liquid nitrogen, and then extracting proteins in $3 \mathrm{ml}$ extraction buffer $(0.1 \mathrm{M}$ Tris- $\mathrm{HCl}$ buffer, $\mathrm{pH}$ 7.0). The suspension was centrifuged and the proteins in the supernatant fraction were precipitated with $80 \%$ saturated ammonium sulfate, collected by centrifugation, and resuspended in $50 \mu \mathrm{l}$ extraction buffer. The sample was then desalted by gel filtration using Sephadex G-25 fine (GE Healthcare, Piscataway, NJ, USA) equilibrated with the extraction buffer. The eluate was used as the crude extract for the enzyme assay. Protein concentrations were quantified using a CBB protein assay kit (Thermo Fisher Scientific Inc., Waltham, MA, USA) with bovine serum albumin as the standard. As leucoanthocyanidins, the reaction products of the DFR reaction, are unstable and hard to detect directly using HPLC, we converted the leucoanthocyanidins to anthocyanidins in a post-enzyme reaction (Shimada et al. 2004, with slight modification). For the DFR enzyme reaction and the conversion to detect the reaction products, $\beta$-NADPH was purchased from Oriental Yeast Co., Ltd. (Tokyo, Japan), DHK was purchased from Sigma-Aldrich Co., Ltd. (St. Louis, MO, USA), taxifolin (DHQ) was obtained from Wuhan ChemFaces Biochemical Co., Ltd. (Wuhan, China), DHM was obtained from Extrasynthase (Z.I. Lyon Nord, Genay Cedex, France), glucose-6-phosphate dehydrogenase from Leuconostoc mesenteroides (G6PDH) and glucose6-phosphate (G6P) were purchased from Sigma-Aldrich Co., Ltd., and quercetin (Qr) and anthocyanidins (Pg, $\mathrm{Cy}, \mathrm{Dp}$ ) were purchased from Extrasynthase. The mixtures contained $0.1 \mathrm{M}$ Tris- $\mathrm{HCl}$ buffer $(\mathrm{pH} 7.0$ ), $2 \mathrm{mM} \beta$-NADPH, $150 \mu \mathrm{M}$ DHK, 1 unit G6PDH, $6 \mathrm{mM}$ G6P in a final volume of $500 \mu$ l. Reactions were initiated by the addition of $100-200 \mu \mathrm{g}$ of enzyme proteins and terminated by extraction with $500 \mu \mathrm{l}$ of ethyl acetate after incubation at $30^{\circ} \mathrm{C}$ for $4 \mathrm{~h}$. After the incubation, $2 \mathrm{mM}$ Qr was added as an internal standard. The ethyl acetate extracts were evaporated and the residues were dissolved in $20 \mu \mathrm{l}$ of $5 \%$ hydrochloric acid in $n$-butanol followed by boiling for $8 \mathrm{~min}$. The boiled samples were evaporated and residues were dissolved in methanol. The samples were filtered to remove insoluble residues and the filtrates were loaded onto the HPLC. HPLC-DAD analysis was carried out using the equipment described earlier with a reversed-phase column of COSMOSIL $5 \mathrm{C}_{18}$-MS-II (i.d. $4.6 \mathrm{~mm} \times 50 \mathrm{~mm}$, Nacalai Tesque, Inc., Kyoto, Japan). The analysis used linear gradient elution at a flow rate of $1.5 \mathrm{ml} \mathrm{min}^{-1}$ from 35 to $80 \%$ methanol in $1.5 \%$ aqueous phosphoric acid for $5 \mathrm{~min}$.

The dark blue variety 'GB' had an approximately 6-fold higher level of DFR gene expression than the pale blue variety 'PB' (Figure 2A). There was a related 78-fold increase in DFR enzyme activity in the crude extract (Figure 2B) and a 5-fold higher level of anthocyanin in 'GB' than in 'PB' (Figure 2C). In comparison to the pale pink variety 'SKH', $D F R$ expression in the orange variety 'NDC' showed a 4 -fold increase while that in the red variety 'CRD' was increased by 2 -fold. DFR enzyme activities in 'NDC' and 'CRD' were approximately 3- and 2 -fold higher than in 'SKH', while anthocyanin levels showed approximately 13 - and 7 -fold increases. These results suggest that the level of DFR gene transcription is associated with the level of translated DFR protein detected by DFR enzyme activity in the crude extract. Additionally, this association might be one of key steps that regulate the level of synthesis and accumulation of anthocyanins in the sepals of 'GB', 'PB', 'NDC', 'CRD' and 'SKH' varieties, irrespective of the anthocyanin aglycones, Dp and Pg. Differences in the amounts of reaction product of DFR, namely leucoanthocyanidins, might be the cause of the differences in anthocyanin levels. The other pale pink variety 'SHP', showed a similar DFR expression level and DFR enzyme activity as 'NDC', but had a low level of anthocyanin similar to that in 'SKH'. This suggests that other step(s) must also influence color intensity. For example, the dynamics of DFR enzyme activity in each variety, such as higher kinetic turnover rates, might cause more efficient catalysis of substrates.

To investigate the possibility that color intensity differences in delphinium flowers are caused by the dynamics of DFR enzyme activity in each variety, such as higher kinetic turnover rates causing more efficient catalysis of substrates to give higher amounts of anthocyanidins in sepals, we examined the properties of recombinant DFR enzymes. Representative enzymes of each of the two groups were analyzed to calculate $k_{\text {cat }} / K_{\mathrm{m}}$ of the purified recombinant DFR proteins. Fulllength coding regions of DgDFR (represented by 'PB' DFR) and DnDFR (represented by 'NDC' DFR) were amplified from 'PB' and 'NDC' cDNAs, respectively, and introduced into the pGEX-6P-2 vector (Amersham Pharmacia Biotech Inc, Picataway, NJ, USA) including a glutathione $S$-transferase (GST)-tag. Escherichia coli BL21 (DE3) competent cells were transformed with the recombinant plasmid vectors. E. coli cells were cultured 
Table 1. Substrate specificities and kinetic turnover rates of DgDFR and DnDFR.

\begin{tabular}{cccc}
\hline & Substrates & $\begin{array}{c}\mathrm{pmol} \mathrm{s}^{-1} \mathrm{mg}^{-1} \\
\text { (relative activities (\%)) }\end{array}$ & $k_{\text {cat }} / K_{\mathrm{m}}\left(\mathrm{s}^{-1} \mathrm{M}^{-1}\right)$ \\
\hline DgDFR & DHK & $2.8 \pm 0.6(100)$ & $6.1 \pm 0.5$ \\
& DHQ & $0.4 \pm 0.1(14)$ & $2.0 \pm 0.1$ \\
& DHM & $0.4 \pm 0.1(14)$ & $0.5 \pm 0.2$ \\
DnDFR & DHK & $43.1 \pm 3.9(100)$ & $6.0 \pm 1.9$ \\
& DHQ & $10.1 \pm 0.9(23)$ & $2.3 \pm 0.7$ \\
& DHM & $4.9 \pm 0.4(11)$ & $1.6 \pm 0.1$
\end{tabular}

Recombinant DFR enzymes were produced with heterologous expression in $E$. coli and purified by GST affinity column. DFR reaction was performed, followed by conversion from leucoanthocyanidin to anthocyanidin. The converted reaction products were separated by HPLC. The anthocyanidin peak areas were used to estimate the amounts of catalytic products.

in Luria Broth medium containing $50 \mu \mathrm{g}$ ampicillin until $\mathrm{OD}_{600}=0.5-0.6$ at $30^{\circ} \mathrm{C}$; then DgDFR or DnDFR protein production was induced by the addition of $1 \mathrm{mM}$ isopropyl $\beta$-D-1-thiogalactopyranoside and the cells were cultured for a further $24 \mathrm{~h}$ at $16^{\circ} \mathrm{C}$. The E. coli cells were harvested by centrifugation, followed by washing with $0.1 \mathrm{M}$ potassium phosphate buffer $(\mathrm{KPi}), \mathrm{pH} 7.5$. The cell pellet was suspended in $0.1 \mathrm{M} \mathrm{KPi}$, pH 7.5, containing $150 \mathrm{mM} \mathrm{NaCl}$, and sonicated on ice. Cell debris was removed by centrifugation and the supernatant was loaded onto a GST affinity column (GSTrap HP $5 \mathrm{ml}$, GE Healthcare) followed by washing and elution according to the manufacturer's protocol. The GST-tag DFR fusion proteins in the eluates were concentrated by centrifugation using an Amicon Ultra-15 centrifuge (50,000 Da molecular weight cut-off, Merck Millipore, Billerica, MA, USA). Protein purity was examined by SDS-polyacrylamide gel electrophoresis (Supplementary Figure S3). The amounts of proteins were quantified as described above. The mixtures contained $0.1 \mathrm{M}$ Tris- $\mathrm{HCl}$ buffer, $\mathrm{pH}$ 7.0, $2 \mathrm{mM} \beta$-NADPH, DHK or DHQ or DHM in the range 3.75 to $600 \mu \mathrm{M}$ in a $500 \mu \mathrm{l}$ final volume. Reactions were initiated by the addition of $50-80 \mu \mathrm{g}$ of enzyme proteins and terminated by extraction with $500 \mu \mathrm{l}$ ethyl acetate after incubation at $30^{\circ} \mathrm{C}$ for $1 \mathrm{~h}$. The conditions for conversion of leucoanthocyanidin and the detection of anthocyanidin by HPLC were as described above.

To analyze the effects of differences in the enzymatic properties of DgDFR and DnDFR on flower color intensity, we compared substrate specificities and kinetic turnover rates (Table 1 ). The $k_{\text {cat }} / K_{\mathrm{m}}$ values of DgDFR for the dihydroflavanols DHK, DHQ and DHM were 6.1, 2.0 and 0.5, respectively, and those of DnDFR were 6.0, 2.3 and 1.6, respectively. That is, the substrate specificities of DgDFR and DnDFR were almost identical, and both showed the highest preference for DHK as the substrate. Although DFR in blue flowers might have been expected to show substrate specificity for DHM, the precursor of the blue pigment $\mathrm{Dp}$, our results surprisingly indicated that the DFR in blue flowers of delphinium had substrate specificity for DHK, the precursor of the red pigment Pg. The kinetic turnover rates of DnDFR enzymatic activity for DHK, DHQ and DHM were 15, 25, and 12 times higher, respectively, than those of DgDFR, indicating that the reaction rate of DnDFR was higher than that of DgDFR. The varieties 'SKH' and 'SHP', which have pale colored sepals, showed similarly low kinetic turnover rates for DgDFR, while the varieties 'NDC' and 'CRD', which have deeply colored sepals, had similarly higher kinetic turnover rates for DnDFR. Although the levels of DFR expression (Figure 2A) and enzyme activities in crude extracts of sepals (Figure 2B) were similar in 'SHP' and 'NDC', the levels of anthocyanin in the former was much lower compared to the latter (Figure 2C). Possibly, the lower kinetic turnover rate of DgDFR in 'SHP' might restrict catalytic activity in vivo compared to DnDFR in 'NDC'; this might cause a lower metabolic flow rate in the anthocyanin synthesis pathway and lower levels of anthocyanin in 'SHP', compared to higher synthesis rate and higher anthocyanin levels in 'NDC'.

In conclusion, the color intensity of delphinium sepals was found to depend on DFR expression levels except in the 'SHP' variety. The DFRs of the blue flowering varieties $D$. grandiflorum and the red flowering varieties $D$. nudicaule and $D$. cardinale had different kinetic properties, which might also have contributed to determining color intensity. The pool size and flow rates of metabolic intermediates, especially DHK, will be investigated in future experiments. Since catalytic step(s) in the anthocyanin synthesis pathway in addition to DFR may also possibly be rate limiting, the levels of expression of genes for other enzymes will need to be quantified and their enzyme activities will need to be determined in 'SHP'. Delphinium sepals also accumulate flavonol glycosides and, thus, the possible co-pigmentation roles of flavonoids in modulating flower color hue need to be considered (Özden et al. 1998; Yoshida et al. 2009). Additionally, since visible flower color is affected by cell shape, vacuolar $\mathrm{pH}$, and anthocyanic vacuolar inclusion formation, as well as accumulated anthocyanin molecules, the possibility of multiple factors influencing flower color intensity will be examined in future studies.

\section{Acknowledgements}

This work was supported by Grant-in-Aid for Scientific Research (B) (23370016).

\section{References}

Collette VE, Jameson PE, Schwinn KE, Umaharan P, Davies KM (2004) Temporal and spatial expression of flavonoid biosynthetic genes in flowers of Anthurium andraeanum. Physiol Plant 122: 297-304

Forkmann G, Ruhnau B (1987) Distinct substrate specificity of dihydroflavonol 4-reductase from flowers of Petunia hybrida. $Z$ 
Naturforsch 42c: 1146-1148

Gopaulchan D, Umaharan P, Lennon AM (2014) A molecular assessment of the genetic model of spathe color inheritance in Anthurium andraeanum (Hort.). Planta 239: 695-705

Hashimoto F, Tanaka M, Maeda H, Fukuda S, Shimizu K, Sakata Y (2002) Changes in flower coloration and sepal anthocyanins of cyanic Delphinium cultivars during flowering. Biosci Biotechnol Biochem 66: 1652-1659

Helariutta Y, Elomaa P, Kotilainen M, Seppanen P, Teeri TH (1993) Cloning of cDNA coding for dihydroflavonol 4-reductase (DFR) and characterization of $d f r$ expression in the corollas of Gerbera hybrida var. Regina (Compositae). Plant Mol Biol 22: 183-193

Heller W, Forkmann G, Britsch L, Grisebach H (1985) Enzymatic reduction of $(+)$-dihydroflavonols to flavan-3,4-cis-diols with flower extracts from Matthiola incana and its role in anthocyanin biosynthesis. Planta 165: 284-287

Johnson ET, Ryu S, Yi H, Shin B, Cheong H, Choi G (2001) Alteration of a single amino acid changes the substrate specificity of dihydroflavonol 4-reductase. Plant J 25: 325-333

Johnson ET, Yi H, Shin B, Oh BJ, Cheong H, Choi G (1999) Cymbidium hybrid dihydroflavonol 4-redectase does not efficiently reduce dihydrokaempferol to produce orange pelargonidin-type anthocyanins. Plant J 19: 81-85

Katsumoto Y, Fukuchi-Mizutani M, Fukui Y, Brugliera F, Holton TA, Karan M, Nakamura N, Yonekura-Sakakibara K, Togami J, Pigeaire A, et al. (2007) Engineering of the rose flavonoid biosynthetic pathway successfully generated blue-hued flowers accumulating delphinidin. Plant Cell Physiol 48: 1589-1600

Kondo T, Oki K, Yoshida K, Goto T (1990) Structure of violdelphin, an anthocyanin from violet flower of Delphinium hybridum. Chem Lett 19: 137-138

Kondo T, Suzuki K, Yoshida K, Oki K, Ueda M, Isobe M, Goto T (1991) Strucuture of cyanodelphin, a tetra-p-hydroxybenzoated anthocyanin from blue flower of Delphinium hybridum. Tetrahedron Lett 44: 6375-6378

Mato M, Onozaki T, Ozeki Y, Higeta D, Itoh Y, Yoshimoto Y, Ikeda H, Yoshida H, Shibata M (2000) Flavonoid biosynthesis in whiteflowered Sim carnations (Dianthus caryophyllus). Sci Hortic (Amsterdam) 84: 333-347

Meyer P, Heidmann I, Forkmann G, Saedler H (1987) A new petunia flower colour generated by transformation of a mutant with a maize gene. Nature 330: 677-678

Miyagawa N, Nishizaki Y, Miyahara T, Okamoto M, Hirose Y,
Ozeki Y, Sasaki N (2014) Sequence variations in the flavonoid $3^{\prime}, 5^{\prime}$-hydroxylase gene associated with reddish flower phenotypes in three delphinium varieties. Plant Biotechnol 31: $83-87$

Mol J, Cornish E, Mason J, Koes R (1999) Novel colored flowers. Curr Opin Biotechnol 10: 198-201

Nishihara M, Nakatsuka T (2011) Genetic engineering flavonoid pigments to modify flower color in floricultural plants. Biotechnol Lett 33: 433-441

Nishizaki Y, Matsuba Y, Okamoto E, Okamura M, Ozeki Y, Sasaki N (2011) Structure of the acyl-glucose-dependent anthocyanin 5-O-glucosyltransferase gene in carnations and its disruption by transposable elements in some varieties. Mol Genet Genomics 286: 383-394

Nishizaki Y, Yasunaga M, Okamoto E, Okamoto M, Hirose Y, Yamaguchi M, Ozeki Y, Sasaki N (2013) p-Hydroxybenzoylglucose is a Zwitter donor for the biosynthesis of 7-polyacylated anthocyanin in delphinium. Plant Cell 25: 4150-4165

Özden S, Dürüst N, Toki K, Saito N, Honda T (1998) Acylated kaempferol glycosides from the flowers of Delphinium formosum. Phytochemistry 49: 241-245

Shimada S, Takahashi K, Sato Y, Sakuta M (2004) Dihydroflavonol 4-reductase cDNA from non-anthocyanin-producing species in the Caryophyllales. Plant Cell Physiol 45: 1290-1298

Stafford HA, Lester HH (1982) Enzymic and nonenzymic reduction of (+)-dihydroquercetin to its 3,4-diol. Plant Physiol 70: 695-698

Stafford HA, Lester HH (1985) The conversion of (+)dihydromyricetin to its 3,4-diol (leucodelphinidin) and to $(+)$ gallocatechin by reductases extracted from tissue cultures of Gingo biloba and Pseudotsuga menziesii. Plant Physiol 70: 695-698

Tanaka Y, Burglira F (2013) Flower colour and cytochromes P450. Phil Trans R Soc B 368: 20120432

Tanaka Y, Tsuda S, Kusumi T (1998) Metabolic engineering to modify flower color. Plant Cell Physiol 39: 1119-1126

Yan F, Di S, Rodas FR, Torrico TR, Murai Y, Iwashina T, Anai T, Takahashi R (2014) Allelic variation of soybean flower color gene W4 encoding dihydroflavonol 4-reductase 2. BMC Plant Biol 14: 58

Yoshida K, Mori M, Kondo K (2009) Blue flower color development by anthocyanins: from chemical structure to cell physiology. Nat Prod Rep 26: 884-915 